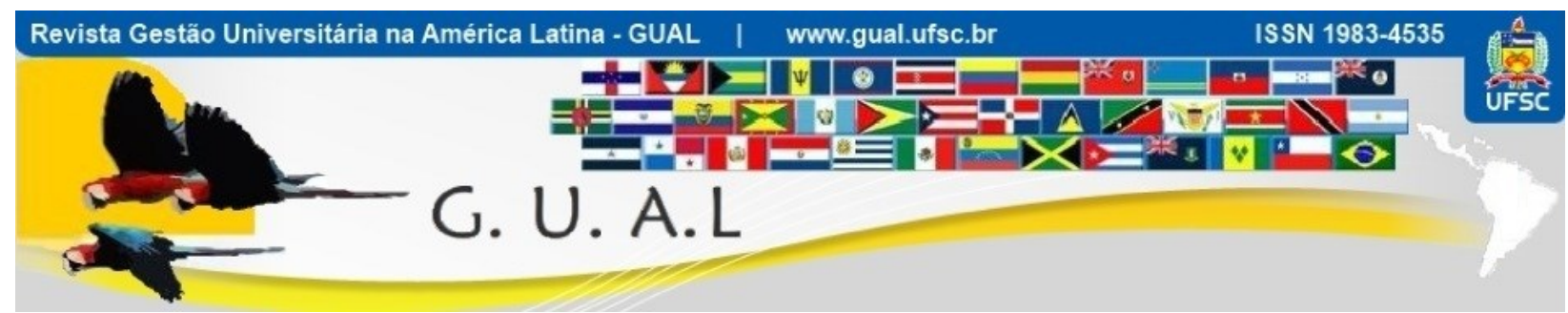

DOI: http://dx.doi.org/10.5007/1983-4535.2015v8n3p114

\title{
EVIDENCIAÇÃO DO ESTADO DA ARTE DO TEMA GESTÃO DE DOCENTES EM INSTITUIÇÕES DE ENSINO SUPERIOR
}

\section{MANAGEMENT OF INSTRUCTORS IN HIGHER EDUCATION INSTITUTIONS: DISCLOSURE OF THE STATE OF THE ART}

Leonardo Ensslin, PhD

Universidade do Sul de Santa Catarina - UNISUL

leonardoensslin@gmail.com

Ademar Dutra, Pós-Doutor Universidade do Sul de Santa Catarina - UNISUL ademar.unisul@gmail.com

Maurício Andrade de Lima, Doutor Universidade do Sul de Santa Catarina - UNISUL mauricio.lima@unisul.br

Caroline Carneiro, Mestranda Universidade do Sul de Santa Catarina - UNISUL carolinecarneirofln@,gmail.com

Leonardo Corrêa Chaves, Doutorando Universidade Federal de Santa Catarina - UFSC leonardomg@gmail.com

Recebido em 09/fevereiro/2014

Aprovado em 02/julho/2015

Sistema de Avaliação: Double Blind Review

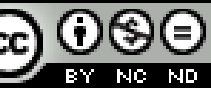

Esta obra está sob uma Licença Creative Commons Atribuição-Uso. 


\title{
RESUMO
}

O objetivo desta pesquisa é aprofundar o conhecimento dos pesquisadores sobre o tema Gestão de Docentes em Instituições de Ensino Superior, com o intuito de conhecer as mais relevantes produções científicas internacionais sobre o assunto, seus autores, periódicos e palavras-chave. Para tanto, foi elaborado um Portfólio Bibliográfico, utilizando-se como instrumento de intervenção o ProKnow-C que, após pesquisa nas bases Scopus, Web of Science, ScienceDirect, ASSIA e Ebsco host, e o processo de filtragem realizado com o auxílio do instrumento de intervenção, resultou em 18 artigos finais. A partir deste portfólio foi possível evidenciar que o periódico mais presente na literatura é o Teaching and Teacher Education. $\mathrm{O}$ artigo mais citado é o The impact of training of university teachers on their Os autores com mais destaque são: Lindblom-Ylänne, S., Nevgi, A. e Trigwell, K. e, as palavraschave mais presentes são: Teacher, University, Performance, Evalu, Management, Assess e Higher Education.

Palavras-chave: Gestão de desempenho. Instituições. Docentes. Avaliação.

\begin{abstract}
The objective of this research is to deepen the knowledge of the researchers on the topic Teachers Management in Higher Education Institutions in order to meet the most relevant international scientific publications on the subject, authors, journals and keywords. Thus, a Library Portfolio was developed, using as an intervention tool the ProKnow-C, after research in Scopus databases, Web of Science, ScienceDirect, ASSIA and Ebsco Host, and the filtering process carried out with the aid of the instrument intervention, resulted in 18 final articles. From this portfolio was possible to show that the more present in the literature is the journal Teaching and Teacher Education. The most cited article is The impact of training of university teachers on Their Authors are more prominently: Lindblom-Ylänne, S., Nevgi, A. and Trigwell, K., and the most present key words are: Teacher, University, Performance, Evalú, Management, Assess and Higher Education.
\end{abstract}

Keywords: Performance Management. Institutions. Teaching. Assessment. 


\section{INTRODUÇÃO}

O ponto de partida para a elaboração de uma pesquisa acerca de um tema específico se dá na construção de uma adequada revisão de literatura, cuja função é apresentar e elucidar o conhecimento científico existente sobre o assunto pesquisado. De acordo com Santos et al. (2006), a geração de conhecimento deve iniciar com a pesquisa do que já foi previamente publicado a respeito do tema estudado.

O conhecimento científico, além de abundante, dissemina-se em uma grande variedade de publicações e fontes de pesquisa, o que acaba tornando-se um grande desafio para quem pretende realizar uma pesquisa sobre determinado tema.

Neste sentido, este trabalho apresenta o processo para selecionar um portfólio bibliográfico alinhado ao tema Gestão de Docentes em Instituições de Ensino Superior e com representatividade acadêmica. Este portfólio bibliográfico, para as delimitações postas pelo pesquisador passa a representar o "estado da arte" ou "estado do conhecimento" no tema como entendido pelo pesquisador e para as delimitações postas pelo mesmo.

Uma vez constituído o referencial bibliográfico a etapa seguinte é a análise e evidenciação dos parâmetros que o pesquisador deseja conhecer. Esta etapa é por vezes denominada bibliometria.

Segundo Guedes, Borschiver (2005) a bibliometria teve suas origens em 1922 com o propósito de esclarecer os processos científicos e tecnológicos, por meio da contagem de documentos. A partir de então houve muitas expansões de seu entendimento e nas últimas décadas pesquisadores como Pao (1992) observaram que um portfólio de artigos pode ser estudado em termos estatísticos. Dentre os parâmetros que podem ser estudados encontramse: periódicos, citações dos artigos, autores de destaque, palavras chave, universidades dentre outros. É dentro desta ideia que se insere a análise bibliométrica a ser realizada nesta pesquisa com o propósito de evidenciar, os mais destacados: periódicos, artigos, autores, universidades e palavras chave, para o tema como o entende o pesquisador.

Dentro deste contexto surge a pergunta para a presente pesquisa: Como realizar a seleção e análise de um referencial teórico de relevância acadêmica e alinhado ao tema singular que se propõe estudar?

O presente artigo busca responder a essa pergunta propondo como objetivo geral selecionar um portfólio bibliográfico sobre o tema: Gestão de Docentes em Instituições de Ensino Superior. Este processo se dá por meio de uma pesquisa em bases de artigos 
científicos disponibilizadas no Portal da CAPES, análise bibliométrica dos artigos relevantes e alinhados ao tema.

Este trabalho está estruturado em seis seções, sendo esta primeira seção de caráter introdutório acerca do tema de pesquisa e os objetivos do trabalho. A segunda, apresenta a metodologia de pesquisa empregada no trabalho em termos do enquadramento metodológico da pesquisa e do instrumento de pesquisa utilizado. A terceira, apresenta o referencial teórico do tema Gestão de Docentes em Instituições de Ensino Superior e da visão de mundo adotada a avaliação de desempenho. A quarta apresenta a aplicação detalhada da metodologia, evidenciando todas as etapas para a obtenção do portfólio bibliográfico, e a análise bibliométrica realizada. A quinta, as considerações finais evidenciando a pergunta de pesquisa e o objetivo e como foram alcançados e os resultados e recomendações para trabalhos futuros, e a sexta, relaciona a bibliografia utilizada.

\section{METODOLOGIA}

Nesta seção, será apresentado o enquadramento metodológico, bem como o instrumento de intervenção utilizado para alcançar os objetivos propostos.

\subsection{ENQUADRAMENTO METODOLÓGICO}

O enquadramento metodológico da pesquisa é apresentado através de seis dimensões: objetivo da pesquisa; natureza da pesquisa; coleta de dados; abordagem do problema; resultados e instrumento de intervenção.

Esta pesquisa, quanto ao seu objetivo, caracteriza-se como exploratória/descritiva, por proporcionar uma investigação sobre um tema específico, permitindo uma visão mais ampla sobre o assunto estudando as características específicas de um determinado grupo de variáveis. (RICHARDSON, 2008).

Pela sua natureza, caracteriza-se como teórica-ilustrativa porque apresenta um processo para realização da busca bibliográfica com ênfase no tema proposto pelo pesquisador, agregado à aplicação do processo de análise bibliométrica (ALAVI \& CARLSON, 1992).

A lógica da pesquisa é indutiva por realizar a observação de informações de um problema cujo conhecimento se pretende adquirir e explicitá-lo durante o decorrer do trabalho (GIL, 2008). 
Para a coleta de dados foram utilizados dados primários e secundários. Os dados primários resultaram das delimitações estabelecidas pelos pesquisadores ao longo do processo de seleção do portfólio bibliográfico; e os dados secundários, se deram a partir das análises dos artigos do portfólio bibliográfico.

Quanto à abordagem do problema, caracteriza-se como pesquisa qualitativa devido ao processo desenvolvido para identificar os artigos do portfólio bibliográfico e suas referências e quantitativa, em função das análises realizadas por meio de contagem das variáveis investigadas (RICHARDSON, 1999).

O resultado da pesquisa é de natureza aplicada, uma vez que permite que o conhecimento adquirido seja utilizado para a realização dos objetivos específicos (RICHARDSON, 2008). A pesquisa bibliográfica foi utilizada como procedimento técnico pelo fato de compor um arcabouço teórico (GIL, 2008).

O instrumento de intervenção utilizado para a elaboração do Portfólio Bibliográfico foi o ProKnow-C - Knowledge Development Process-Constructivist (Proknow-C), proposto por Ensslin e Ensslin (2007) e Ensslin et al. (2010).

\section{REFERENCIAL TEÓRICO}

A crescente expansão do nível de qualificação profissional exigido pelas empresas associado às motivações para ascender socialmente das classes dos estratos econômicos mais baixos tem feito com que o número de pessoas em busca de instrução superior cresça em taxas muito superiores ao observado no passado. Constatadas as crescentes demandas as instituições de ensino igualmente se multiplicaram e passaram a buscar formas de se qualificar para atrair os melhores alunos. Nesta busca por aperfeiçoamentos estão: a infraestrutura; a qualificação docente; os resultados; dentre outros. A infraestrutura por ser uma propriedade eminentemente objetiva tem sido administrada com objetividade e, portanto facilidade. Já a qualificação docente e os resultados da capacitação dos alunos por envolver a percepção subjetiva dos atores, objetivos conflitantes e, portanto alta complexidade tem encontrado mais dificuldade em seu gerenciamento. Estes condicionantes têm feito com que o entendimento deste contexto tenha tido tantas definições quantos são seus avaliadores. Assim para efeito deste trabalho vamos contextualizar mais uma visão de mundo do contexto referente à gestão dos docentes de IES, conforme o entendimento dado pelo pesquisador a seguir. 
A expansão da educação superior, cada vez mais acelerada em função dos avanços tecnológicos e da atual sociedade do conhecimento, tem sua qualidade diretamente relacionada aos resultados da aprendizagem dos alunos. O processo de transferência do conhecimento se dá através da interação entre alunos e docentes e, para isto, é necessário que o docente possua não só habilidades técnicas, mas também, um método de ensino capaz de estabelecer conexões entre teoria e prática, capacidade de inspirar seus alunos a querer aprender mais, propiciando um ambiente colaborativo em sala de aula. O papel do professor é vital para o sucesso do processo de ensino e aprendizagem nas instituições e, por este motivo, torna-se extremamente necessário acompanhar e orientar seu planejamento e avaliar as suas atividades a fim de que se possa desenvolver ações de melhoria em suas práticas de ensino.

A partir deste entendimento foram identificados que as áreas de conhecimento necessárias para o processo de gestão dos docentes, são: Avaliação de desempenho; Docentes e Universidades. Estas áreas de conhecimento a partir de agora serão denominadas Eixos de Pesquisa e seu entendimento será dados pelas palavras chave que as representam conforme apresentado na Tabela 1.

\begin{tabular}{|c|l|}
\hline Eixos de Pesquisa & \multicolumn{1}{c|}{ Palavras-chave } \\
\hline Avaliação de Desempenho & Appraisal, Assessment, Evaluation, Management, Measurement, Performance. \\
\hline Docentes & Educator, Instructor, Master, Teacher. Trainer. \\
\hline Universidades & College, Education Institution, Higher Education, Private Organization, University. \\
\hline
\end{tabular}

Tabela 1 Eixos de Pesquisa e suas palavras chave

Fonte: Autores da pesquisa (2014)

Vários autores tem contribuído com estudos sobre gestão de docentes no ensino superior na literatura internacional, como forma de aprofundar as discussões sobre o tema e, em seus estudos, abordam os mais diversos fatores de impacto no processo.

A qualidade do ensino como forma de melhorar o desempenho das instituições é tema dos estudos de Brown (2001), Huang e Lin (2014) e Megnounif et al (2013), dentre outros.

Já Castle et al (2006), Day et al (2005), Gibbs e Coffey (2004) e Stewart (2014 focam seus estudos sobre a formação dos docentes no ensino superior, evidenciando a importância de preparar estes profissionais desenvolvendo não só suas habilidades técnicas mas, auxiliando no desenvolvimento de sua liderança e controle emocional. 
Outros pesquisadores, como Henderson et al (2014), Page (2013), Parpala et al (2007) e Rasheed et al (2011) Tejedor et al (2008) abordam o recrutamento, seleção e avaliação no ensino superior, enfatizando a importância do feedback dos alunos na gestão dos docentes.

A presente pesquisa identificou através dos periódicos, artigos, autores e palavraschave existentes na literatura internacional os estudos realizados sobre o tema "gestão de docentes no ensino superior", sob a ótica da Avaliação de Desempenho utilizando o processo ProKnow-C para selecionar um portfólio bibliográfico e realizar sua bibliometria.

\section{RESULTADOS}

Esta seção apresenta os resultados da pesquisa que, através da seleção de artigos, deram origem ao Portfólio Bibliográfico e à sua Bibliometria. Este processo representa o conhecimento construído sobre o tema Gestão de Docentes no Ensino Superior, segundo o entendimento do tema pelo pesquisador.

\subsection{SELEÇÃO DO PORTFÓLIO BIBLIOGRÁFICO}

Essa etapa inicial visa a elaboração de um portfólio bibliográfico que permite ao pesquisador reunir uma gama de artigos relacionados ao seu tema da pesquisa, alinhados de acordo com a sua percepção do tema e com as suas delimitações. São realizadas, nesta etapa, três procedimentos: (a) a seleção dos artigos nas bases de dados que irão compor o Banco de Artigos Bruto; (b) a filtragem dos artigos selecionados com base no alinhamento da pesquisa; e, (c) o teste de representatividade do portfólio bibliográfico. O produto final dessa etapa da pesquisa é um conjunto de artigos que o pesquisador considerou relevante e alinhado com a sua pesquisa, denominado de Portfólio Bibliográfico (PB). Cabe ressaltar que os procedimentos realizados nesta pesquisa ocorreram entre julho e agosto de 2014 .

\subsubsection{Seleção do Banco de Artigos Bruto}

Para dar início ao processo de seleção do Banco de Artigos Bruto foi necessário que os pesquisadores definissem as áreas de conhecimento que norteariam a pesquisa, partindo da sua percepção em relação ao tema. As áreas de conhecimento são definidas aqui como eixos de pesquisa e, para este estudo, foram definidos os seguintes: Avaliação de desempenho, Docentes e Universidades. 
O primeiro eixo, Avaliação de desempenho, está relacionado ao processo de apoio à decisão, o segundo e o terceiro eixos estão diretamente relacionados ao tema em estudo. Assim, os eixos auxiliam os pesquisadores no direcionamento da construção do conhecimento necessário sobre o tema.

Após a definição dos eixos, os pesquisadores partem para a formação do Banco de Artigos Bruto, que é composta por quatro fases distintas, conforme apresentado na Figura 1:

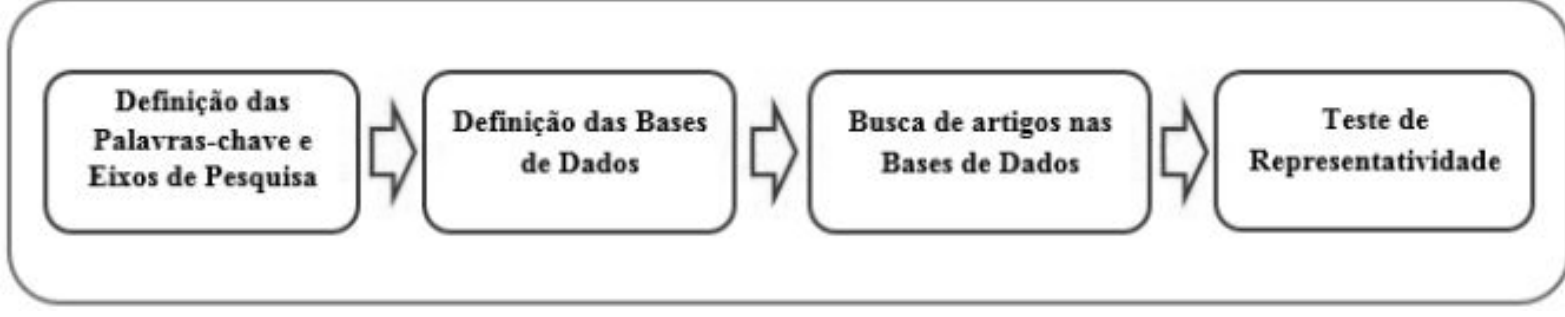

Figura 1 Etapa de Seleção do Banco de Artigos Bruto

Fonte: Autores da Pesquisa (2014)

\section{Definição de palavras-chave}

Para cada eixo de pesquisa foram definidas palavras-chave, que representam o entendimento que os autores definiram para as áreas de conhecimento de seu tema. Para o Eixo 1, Avaliação de Desempenho, foram definidas as seguintes palavras-chave: Measur*, Performance, Assess*, Evalu*, Appraisal e Management. O eixo 2, Docentes, as palavraschave definidas foram: Instructor, Educator, Master, Teacher e Trainer. Já o eixo 3, Universidades, foi composto pelas palavras: "Education Institution", "Higher Education", "Private organization", College e University. O asterisco, após o radical das palavras, foi utilizado aqui com o intuito de que a pesquisa alcançasse as variações verbais ou substantivas das palavras.

\section{Definição das bases de dados}

Após a definição dos eixos e palavras-chave, devem ser escolhidas as bases de dados consideradas potencialmente importantes para a pesquisa, entre as bases disponíveis no Portal de Periódicos da CAPES, identificando as devidamente alinhadas com a área de conhecimento, neste caso, a área de Ciências Sociais Aplicadas.

Para a realização desta pesquisa, foram selecionadas cinco bases de dados que indexaram os periódicos científicos mais aderentes ao tema da pesquisa, são elas: ASSIA, EBSCOhost, ScienceDirect, Scopus e Web of Science. 
A partir da definição das bases, inicia-se um processo de busca, utilizando-se as combinações das palavras-chave definidas pelos eixos de pesquisa (expressão booleana), restringindo-se aos campos de títulos dos artigos (article title), palavras-chave (key words) e resumos (abstracts). A esses critérios de restrição, estabeleceu-se um período temporal de 14 anos, de 2000 a 2014, de trabalhos publicados apenas em periódicos.

Esta busca resultou em 3.379 artigos, conforme apresentado na tabela abaixo:

\begin{tabular}{|cc|}
\hline Base de Dados & Quantidade de Artigos \\
\hline Scopus & 277 \\
Web of Science & 2566 \\
ScienceDirect & 310 \\
ASSIA & 149 \\
EBSCOhost & 77 \\
\hline
\end{tabular}

Tabela 2 Artigos Brutos Selecionados por Base de Dados Fonte: Autores da Pesquisa (2014)

\section{Busca dos artigos nos bancos de dados com as palavras-chave}

A partir das combinações entre as palavras-chave dos três eixos, foi elaborada a "Expressão Booleana de Busca", para ser utilizada na busca dos artigos científicos nas bases de publicações disponibilizadas pela CAPES. A "Expressão Booleana de Busca", está representada na Figura 2.

\section{(Measur * or Performance or Assess* or Evalu*or Appraisal or Management) and (Instructor or Educator or Master or Teacher or Trainer) and ("Education Institution" or "Higher Education" or "Private organization" or College or University)}

Figura 2 Expressão Booleana Palavras-chave Fonte: Autores da Pesquisa (2014)

Para armazenar e organizar os artigos selecionados, foi utilizado o gerenciador bibliográfico EndNote X7, um software especializado em armazenar, gerenciar e procurar referências bibliográfica em sua biblioteca privada de referências. 


\section{Teste de aderência das palavras-chave}

Com o portfólio bruto selecionado, foi realizado o teste de aderência das palavraschave. Este processo se deu através da escolha de dois artigos aleatórios, com o objetivo de identificar se há ou não a necessidade de incluir novas palavras-chave ao portfólio. Para este estudo, após a execução deste processo, concluiu-se que não seria necessária a inclusão de novas palavras-chave, o que indicou o alinhamento destas e dos artigos ao tema da pesquisa. Após a finalização desta fase, iniciou-se o processo de filtragem do Banco de Artigos Brutos.

\subsubsection{Filtragem do Banco de Artigos Brutos}

O processo de filtragem do banco de artigos brutos compreendeu seis etapas:1 Filtragem do Banco de Artigos Brutos quanto à Redundância; 2 - Filtragem quanto ao alinhamento dos títulos dos artigos; 3 - Filtragem quanto ao reconhecimento científico; 4 Filtragem quanto ao alinhamento do resumo ao tema; 5 - Filtragem quanto ao alinhamento íntegral dos artigos ao tema; e, 6 - Teste de Representatividade do Portfólio Bibliográfico. Ao final deste processo de filtragem, estará definido o Portfólio Bibliográfico Final.

A etapa de Filtragem do Banco de Artigos Brutos, pode ser verificada na figura abaixo:

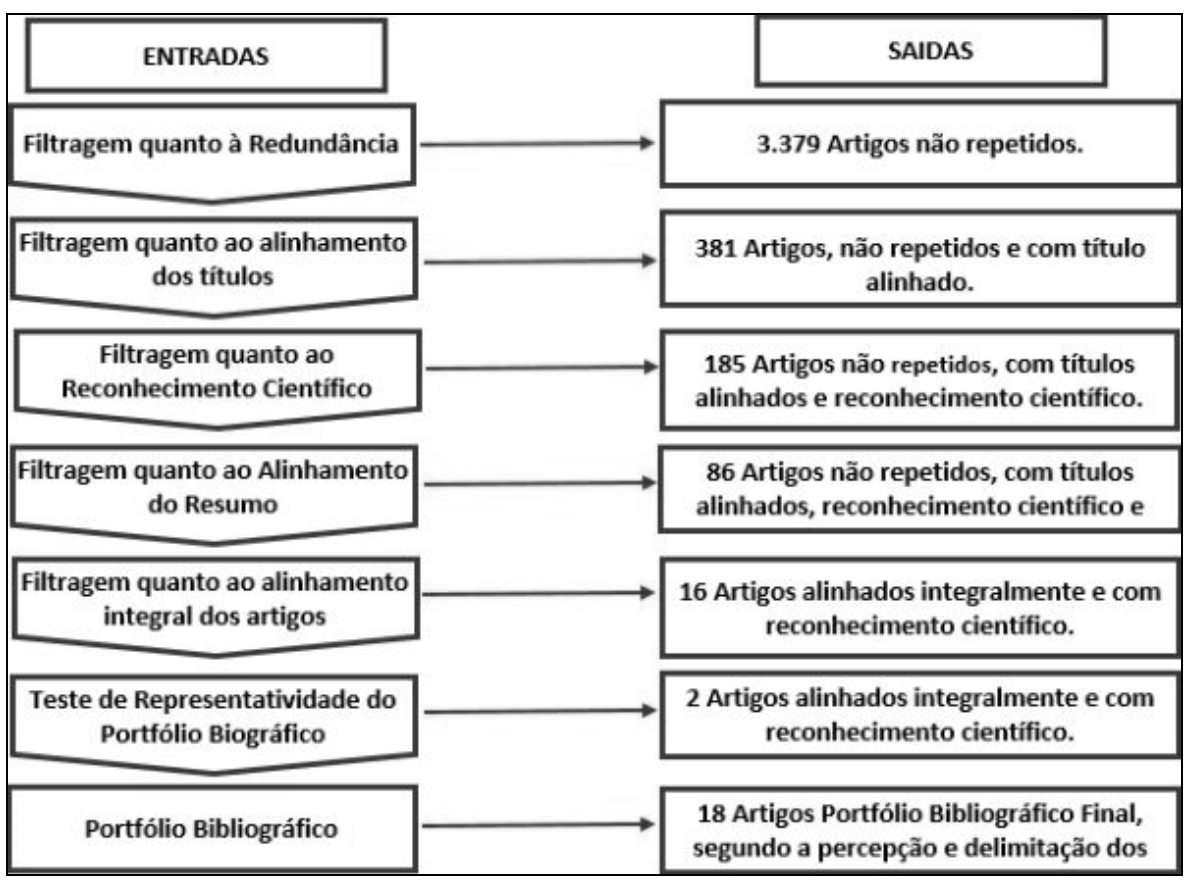

Figura 3 Filtragem do Banco de Artigos Brutos Fonte: Autores da Pesquisa 
Dos 16 artigos do PB na etapa "Filtragem quanto ao alinhamento integral dos artigos", foram identificados 115 artigos denominados Artigos das Referências do Portfólio Bibliográfico que, após serem submetidos a todo o processo de filtragem, resultaram na identificação de dois artigos alinhados ao tema. Estes, foram integrados ao Portfólio Bibliográfico Final $(\mathrm{PB})$ representado na Tabela 3.

Portfólio Bibliográfico - PB

\begin{tabular}{|c|c|}
\hline 1 & $\begin{array}{l}\text { BROWN, W. O. Sources of funds and quality effects in higher education. Economics of Education Review } 20 \\
\text { (2001) 289-295. Accepted } 21 \text { January } 2000 .\end{array}$ \\
\hline 2 & $\begin{array}{l}\text { CASTLE, S.; FOX, R. K.; SOUDER, K. O'H. Do Professional Development Schools (PDSs) make a difference?: } \\
\text { A comparative study of PDS and non-PDS teacher candidates. Journal of Teacher Education, Vol. } 57 \text {, No. } 1 \text {, } \\
\text { January/February } 2006 \text { 65-80. }\end{array}$ \\
\hline 3 & $\begin{array}{l}\text { DAY, C.; ELLIOT, B.; KINGTON, A. Reform, standards and teacher identity: Challenges of sustaining } \\
\text { commitment. Teaching and Teacher Education } 21 \text { (2005) 563-577. }\end{array}$ \\
\hline 4 & $\begin{array}{l}\text { EVANS, L.; HOMER, M.; RAYNER, S. Professors as Academic Leaders: The Perspectives of "the Led". } \\
\text { Educational Management Administration \& Leadership } 2013 \text { 41: } 674 .\end{array}$ \\
\hline 5 & $\begin{array}{l}\text { GIBBS, G., \& COFFEY, M. The impact of training of university teachers on their teaching skills, their } \\
\text { approach to teaching and the approach to learning of their students. Active Learning in Higher Education 1, } \\
\text { Mar } 2004 \text {. }\end{array}$ \\
\hline 6 & $\begin{array}{l}\text { HENDERSON, C.; TURPEN, C.; DANCY, M.; CHAPMAN, T. Assessment of teaching effectiveness: Lack of } \\
\text { alignment between instructors, institutions, and research recommendations. Physical Review Tópicos } \\
\text { Especiais. } 19 \text {, February, } 2014 \text {. }\end{array}$ \\
\hline 7 & $\begin{array}{l}\text { HUANG, Y.-C., LIN, S.-H. (2014). Assessment of Charisma as a Factor in Effective Teaching. Educational } \\
\text { Technology \& Society, } 17 \text { (2), 284-295, } 4 \text { June, } 2013 .\end{array}$ \\
\hline 8 & $\begin{array}{l}\text { KENNEDY, M. M. Knowledge and vision in teaching. Journal of Teacher Education, Vol. 57, No. 3, May/June } \\
2006 \text { 205-211. }\end{array}$ \\
\hline 9 & $\begin{array}{l}\text { LABAREE, D. F. On the nature of teaching and teacher education: Difficult practices that look Easy. Journal } \\
\text { of Teacher Education 1, May } 2000 .\end{array}$ \\
\hline 10 & $\begin{array}{l}\text { MEGNOUNIF, A.; KHERBOUCHE, A.; CHERMITTI, N. Contribution to the Quality Assessment in Higher } \\
\text { Education_The Case Study of the Faculty of Technology, Tlemcen, Algeria. Procedia - Social and Behavioral } \\
\text { Sciences (IFEE 2012). }\end{array}$ \\
\hline 11 & $\begin{array}{l}\text { PAGE, D. Recruitment and Transition of Construction Lecturers in Further Education: The Perspective of } \\
\text { Middle Managers. Educational Management Administration \& Leadership } 2013 \text { 41: 819, originally published } \\
\text { online 11, July 2013. }\end{array}$ \\
\hline 12 & $\begin{array}{l}\text { PARPALA, A.; YLÄNNE, L. S. University Teachers' Conceptions Of Good Teaching In The Units Of High- } \\
\text { Quality Education. Studies in Educational Evaluation } 33(2007) 355-370 .\end{array}$ \\
\hline 13 & $\begin{array}{l}\text { RASHEED, M. I.; ASLAM, H. D.; YOUSAF, S.; NOOR, A. A critical analysis of performance appraisal system } \\
\text { for teachers in public sector universities of Pakistan: A case study of the Islamia University of Bahawalpur } \\
\text { (IUB). African Journal of Business Management Vol. 5(9), pp. 3735-3744, } 4 \text { May, } 2011 \text {. }\end{array}$ \\
\hline 14 & $\begin{array}{l}\text { REID, I. C. Reflections on using the Internet for the evaluation of course delivery. University of South } \\
\text { Australia, Underdale Campus, Holbrooks Road, Underdale 5032, Internet and Higher Education. Accepted } 27 \text { July } \\
2001 .\end{array}$ \\
\hline 15 & $\begin{array}{l}\text { RUEDA B.M.. La Evaluación del Desempeño Docente en la Universidad. The Evaluation of Teacher } \\
\text { Perfomance at the University. Revista electrónica de investigación educativa. Publicado en } 22 \text { de octubre de } 2008 .\end{array}$ \\
\hline 16 & $\begin{array}{l}\text { RYHAMMAR, L.; ANDERSSON, A. L. Relations between university teachers' assessed degree of creativity } \\
\text { and productivity and views regarding their organization. Journal of Creative Behavior. Vol. } 35 \text { Number } 3 \text { Third } \\
\text { Quarter } 2001 .\end{array}$ \\
\hline 17 & $\begin{array}{l}\text { STEWART, M. Making sense of a teaching programme for university academics: Exploring the longer-term } \\
\text { effects. Teaching and Teacher Education, } 38 \text { (2014) } 89 \text { e } 98 \text {. }\end{array}$ \\
\hline 18 & $\begin{array}{l}\text { TEJEDOR T.F.J.; MELIÁ, J. M. J.. University Teacher's Evaluation in Spain. Revista electrónica de } \\
\text { investigación educativa. Publicado em } 15 \text { de octubre de } 2008 .\end{array}$ \\
\hline
\end{tabular}

Tabela 3 Portfólio Bibliográfico

Fonte: Autores da Pesquisa (2014) 
Dos 18 artigos do PB, 11 foram publicados entre 2000 e 2008, 4 artigos de 2011 a 2013 e 3 em 2014, o que mostra que o tema é constantemente pesquisado no meio acadêmico. Os 3 artigos publicados em 2014 são: Assessment of teaching effectiveness: Lack of alignment between instructors, institutions, and research recommendations, que aborda a importância da "Eficácia do Ensino" sob a perspectiva dos métodos de avaliação; o artigo Assessment of Charisma as a Factor in Effective Teaching, também com foco na "Eficácia do Ensino" mas sob a ótica de que o professor, através de suas características e habilidades pode ser o condutor da eficiência no ensino. Já o artigo Making sense of a teaching programme for university academics: Exploring the longer-term effects, com enfoque na qualidade do ensino, aborda a importância do professor no desenvolvimento do ensino.

Através desta análise, das últimas publicações sobre o tema, percebemos que o professor está no centro das pesquisas, que estão analisando não só o seu papel de facilitador no ensino mas, a identificação de suas habilidades, características e motivações.

Este Portfólio Bibliográfico representa o conhecimento construído sobre o tema “Gestão de Docentes no Ensino Superior”, no entendimento dos pesquisadores a partir de sua visão de mundo e da metodologia aqui aplicada. Assim, foi possível conhecer o "estado da arte" sobre o tema, identificando os periódicos, artigos, autores e palavras-chave mais representativos.

Na próxima seção, será apresentada a Análise Bibliométrica que tem como objetivo evidenciar as informações sobre o Portfólio Bibliográfico obtido por meio da análise e quantificação de suas características (Ensslin et al, 2010a).

\subsection{ANÁLISE BIBLIOMÉTRICA}

A Análise Bibliométrica do Portfólio Bibliográfico é realizada para que seja possível visualizar de forma quantitativa os periódicos, artigos, autores e palavras-chave que mais se destacam no tema pesquisado.

\subsubsection{Periódicos de Destaque}

Conforme demonstrado na Figura 4, o periódico de destaque no Portfólio Bibliográfico é o Journal of Teacher Education com um total de 3(três) artigos publicados, já nas suas Referências, o periódico de destaque é o Teaching and Teacher Education, também com 3(três) artigos publicados. 
No Portfólio Bibliográfico, além do periódico Journal of Teacher Education, também merecem destaque os periódicos Educational Management Administration \& Leadership e o Teaching and Teacher Education, ambos com 2(dois) artigos publicados.

Já nas Referências do Portfólio Bibliográfico, além do periódico Journal of Teacher Education, o periódico Active Learning in Higher Education se destaca por ter 2(dois) artigos publicados.

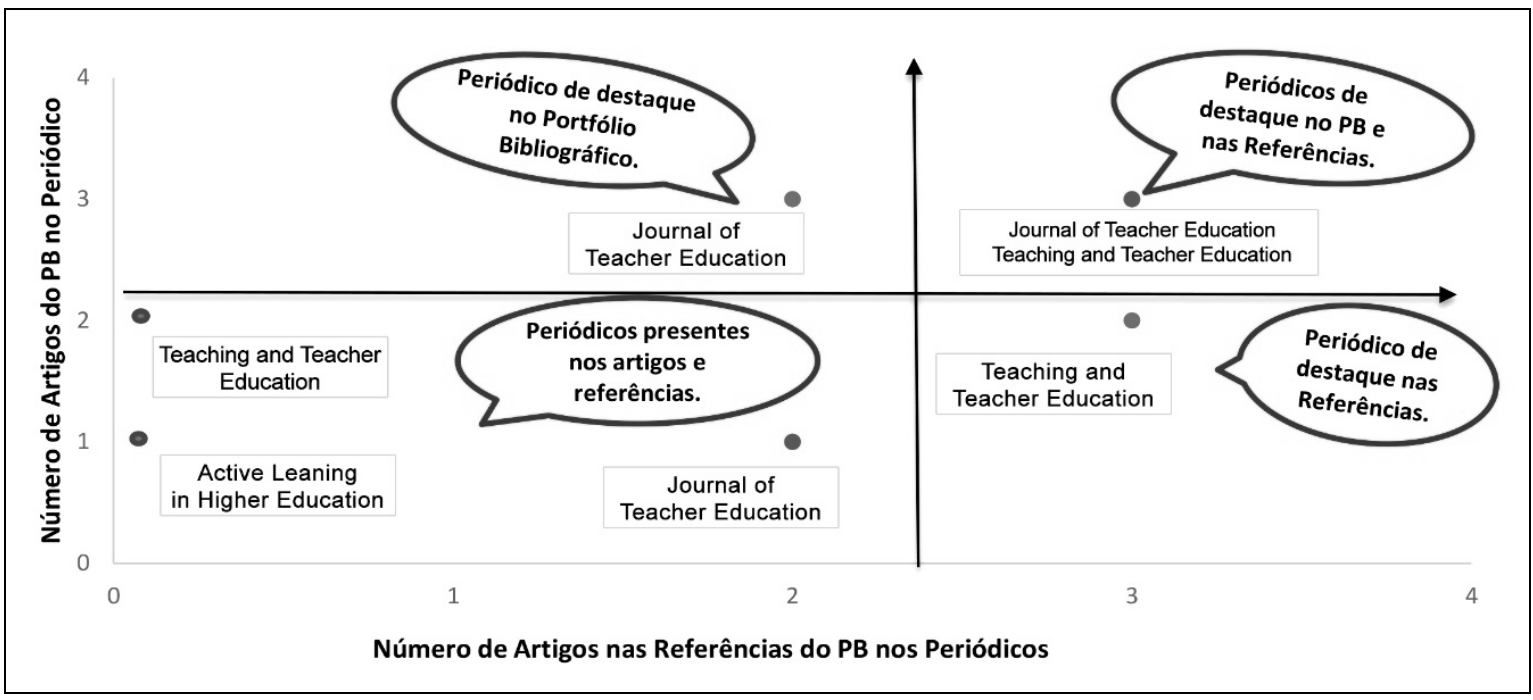

Figura 4 Periódicos de destaque no Portfólio Bibliográfico e nas suas Referências Fonte: Dados da pesquisa - 2014

A partir da análise realizada, constata-se que o periódico com mais destaque no $\mathrm{PB}$ e nas suas Referências é o Teaching and Teacher Education, por constar em 2(dois) artigos do PB 3(três) artigos de suas referências, totalizando 5(cinco) artigos publicados na área relativa ao tema de estudo dos pesquisadores.

Para identificar o fator de impacto dos periódicos na comunidade científica são utilizadas algumas métricas que tem por finalidade identificar os periódicos mais citados em uma determinada área e a relevância da publicação. Neste estudo, foram pesquisados os fatores de impacto dos periódicos nos quais foram publicados os artigos do portfólio bibliográfico através de duas bases - ISI - Web of Knowlegde e Scopus.

Na base ISI - Web of Knowlegde, o fator de impacto dos periódicos é definido pelo indicador JCR - Journal Citation Reports.

Dos 13 periódicos que integram o Portfólio Bibliográfico, apenas 07 apresentam fator de impacto na base ISI. Para uma melhor análise, eles foram subdivididos em três grupos, 
utilizando-se como critério o indicador identificado para cada periódico, conforme apresentado na figura abaixo:

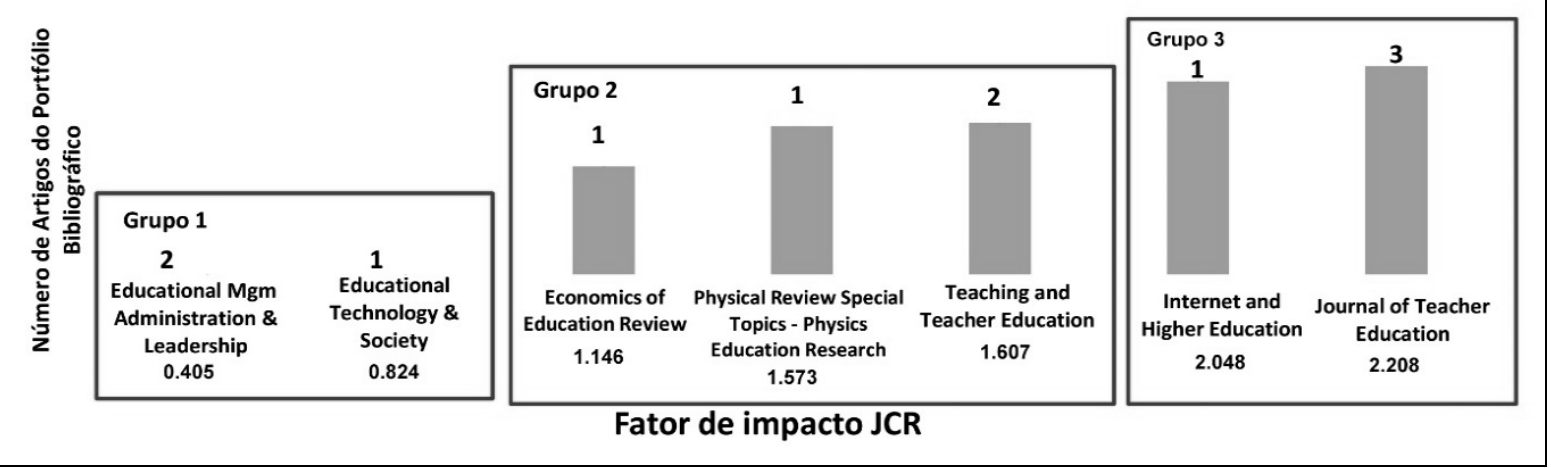

Figura 5 Fator de Impacto dos Periódicos - JCR Fonte: Dados da pesquisa - 2014

O Grupo 1, é composto por dois periódicos que possuem fator de impacto inferior à 1, já o Grupo 2 é composto por três periódicos que apresentam fator de impacto entre 1 e 2, por fim, o Grupo 3 representa os dois periódicos com fator de impacto superior à 2 .

Nesta base, o periódico de destaque é o Journal of Teacher Education, que integra o Grupo 3 pois, além de estar presente em 3 artigos do PB, apresenta o maior fator de impacto, 2.208. Já na base Scopus, o indicador do fator de impacto é o SJR - SCImago Journal Rank que, nesta pesquisa, foi identificado em 11 dos 13 periódicos que integram o PB. Subdividido em três grupos, conforme abaixo, o SJR inferior à 1 foi denominado como Grupo 1, no Grupo 2 estão os periódicos com indicador de 1 até 2 e, no Grupo 3 estão alocados os periódicos com indicador de 2 à 3.

O Grupo 1 destaca-se por apresentar 8 artigos, ou seja, maior frequência de publicações, com fator SJR inferior a 1. Mas, o periódico de destaque é o Journal of Teacher Education com fator de impacto 3.404, conforme apresentado na figura abaixo:

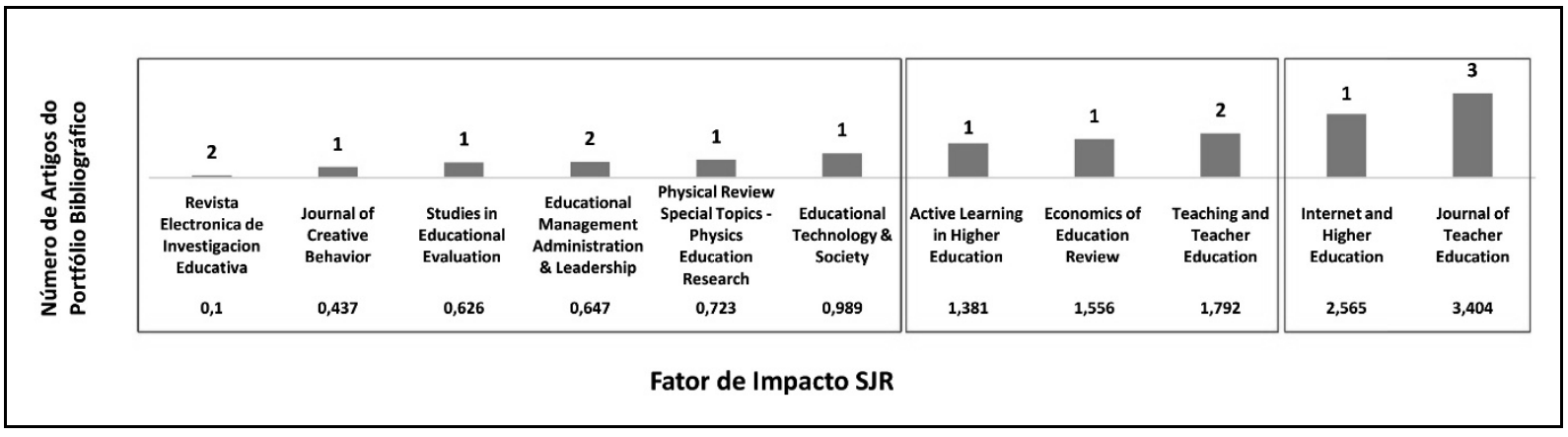

Figura 6 Fator de Impacto dos Periódicos - SJR 
Fonte: Dados da pesquisa - 2014

\subsubsection{Artigos de destaque}

Os artigos do PB foram analisados considerando o número de citações no Google Acadêmico e número de vezes que o autor principal do artigo foi citado nas Referências Bibliográficas do $\mathrm{PB}$. $\mathrm{O}$ artigo The impact of training of university teachers on their teaching skills, their approach to teaching and the approach to learning of their students é o destaque do PB com 444 citações no Google Acadêmico, já o artigo University Teachers' Conceptions Of Good Teaching In The Units Of High-Quality Education destaca-se por ter 2 artigos do seu autor nas referências do PB.

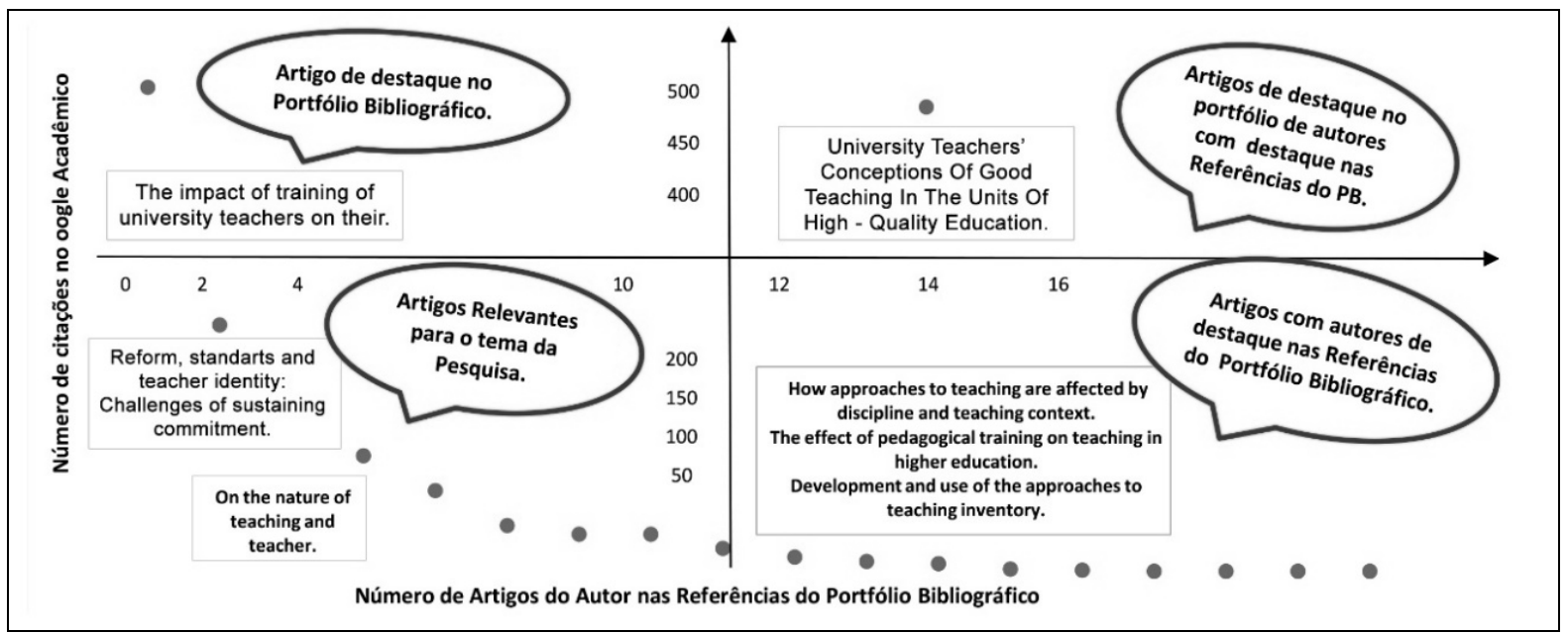

Figura 7 Artigos de destaque no Portfólio Bibliográfico e nas suas Referências Fonte: Dados da pesquisa - 2014

\subsubsection{Autores de destaque}

A Figura 8 apresenta os autores de destaque no tema pesquisado. $\mathrm{O}$ autor LindblomYlänne, S. é destaque com 2 artigos publicados no PB e 1 artigo publicado nas suas Referências.

Também podemos destacar Nevgi, A. e Trigwell, K. com 2 artigos publicados no PB e Gibbs, G. e Labaree, D. F. que aprecem com 1 artigo publicado no PB e 1 nas suas Referências. 


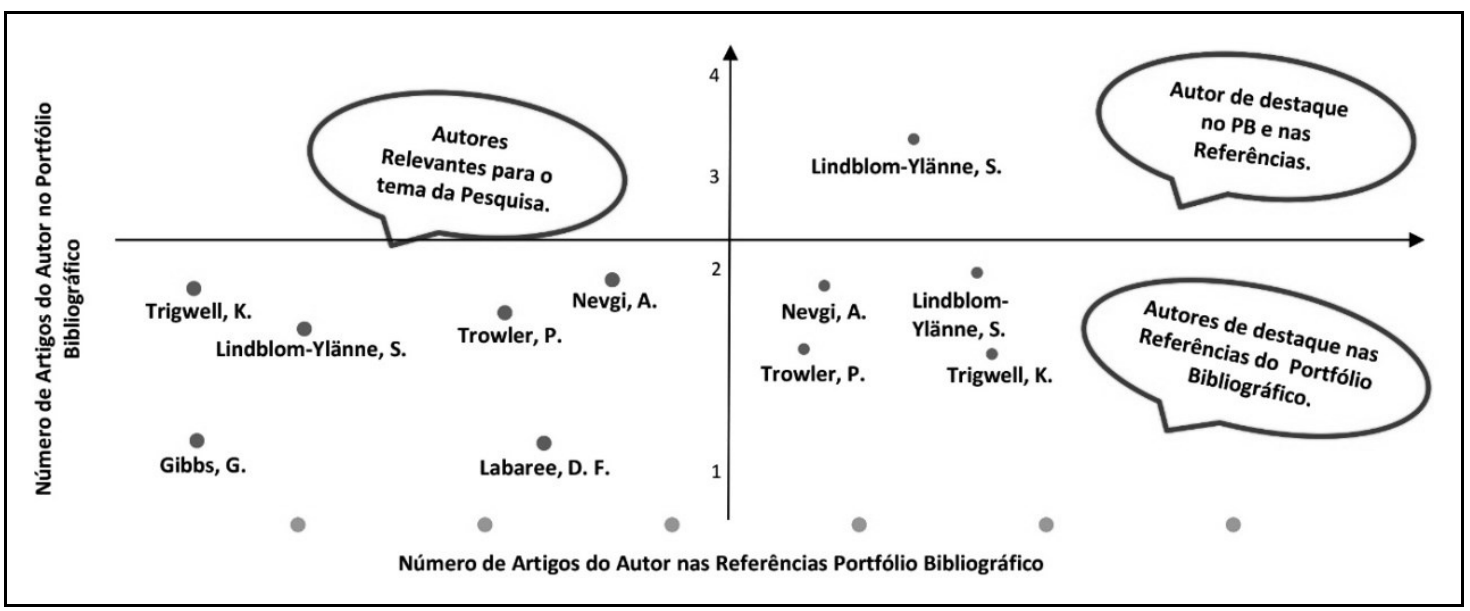

Figura 8 Autores de destaque no Portfólio Bibliográfico e nas suas Referências Fonte: Dados da pesquisa - 2014

\subsubsection{Palavras-chave de destaque}

As palavras-chave foram analisadas, conforme a Figura 9, as que mais se destacaram são as palavras: Teacher com incidência em 17 artigos dos 18 que integram o PB, University com incidência em 16 artigos, Performance, Evalu* e Management que aparecem em 15 artigos.

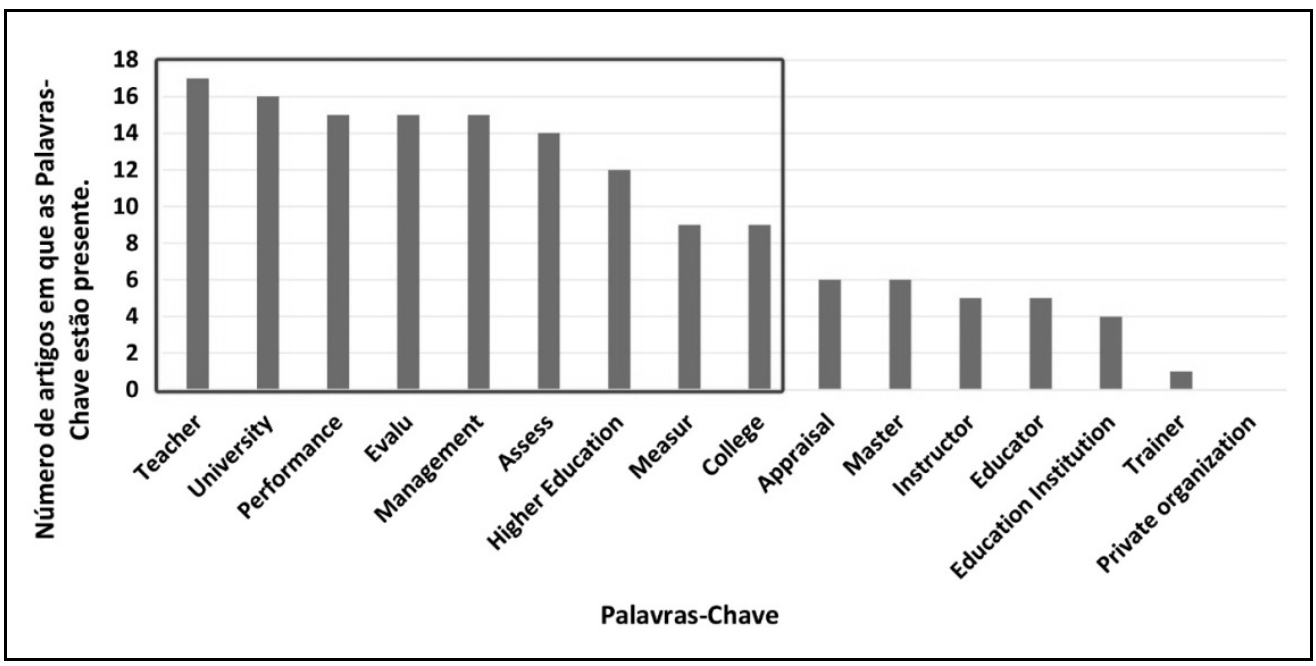

Figura 9: Palavras-chave de destaque no Portfólio Bibliográfico Fonte: Dados da pesquisa - 2014

Está análise demonstra o alinhamento da pesquisa ao tema estudado, uma vez que as palavras-chave previamente definidas pelos pesquisadores, foram identificadas nos artigos do PB e, algumas delas, aparecem em quase $100 \%$ dos mesmos. 


\subsubsection{Universidades de destaque}

$\mathrm{Na}$ análise das Universidades de destaque, ou seja, na identificação de quais Universidades estão os autores do PB, os resultados são apresentados na Figura 10.

O destaque é da Islamia University of Bahawalpur do Paquistão com quatro pesquisadores, e a Faculty of Technology, University of Tlemcen da Argélia também merece destaque pois possuí 3 pesquisadores nos artigos do PB.

Além disso, Estados Unidos e Reino Unido destacam-se como países com maior número de Universidades pesquisando sobre o tema, no total 6 Universidades em cada um.

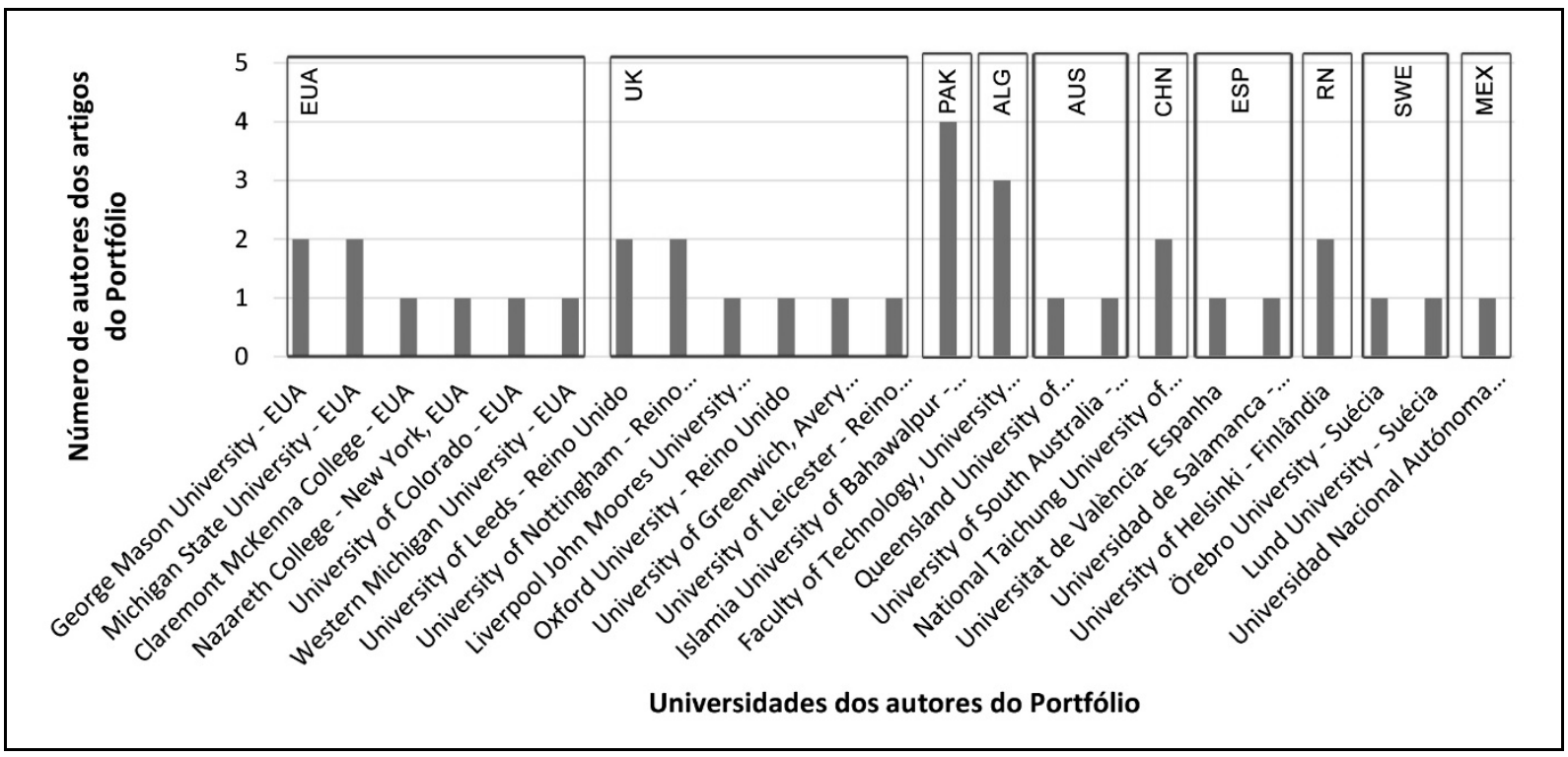

Figura 10 Universidades de destaque

Fonte: Dados da pesquisa - 2014

Podemos perceber que o tema Gestão de Docentes em Instituições de Ensino Superior, objeto de estudo desta pesquisa, é abordado em 23 Universidades localizadas em 10 países diferentes, o que confirma a relevância do assunto para a área acadêmica.

\section{CONSIDERAÇÕES FINAIS}

A universalização do ensino proporcionou o acesso a forma de atuação das instituições que, para manterem-se competitivas no mercado, precisam manter um diferencial. Por ter como foco principal o ensino, este diferencial pode ser percebido na atuação dos seus docentes, por isto, torna-se necessária a utilização de instrumento de gestão que evidencie seus valores e diferenciais competitivos para manter-se no mercado. 
Para compreender o cenário acerca do tema Gestão de Docentes em Instituições de Ensino Superior, se faz necessário responder a pergunta: Como realizar a seleção e análise de um referencial teórico de relevância acadêmica e alinhado ao tema singular que se propõe estudar?

O conhecimento sobre o tema estudado se deu através da elaboração de um Portfólio Bibliográfico, a partir da expansão e consolidação do entendimento dos pesquisadores sobre o tema e, representando: a visão de mundo e delimitações postas pelos mesmos, o portfólio de artigos que evidencia o estado da arte e para esta amostra: os periódicos, artigos, autores e palavras - chave mais representativos.

Este Portfólio Bibliográfico, foi construído utilizando o instrumento de intervenção ProKnow-C que preocupa-se, ao iniciar o processo de seleção, com que o pesquisador tenha bem definido o seu tema e assim, na etapa de seleção do portfólio, concomitantemente com o processo de seleção, o pesquisador tenha oportunidade de conhecer outras pesquisas sobre seu tema e então reveja a definição ou contextualização do mesmo. Há um processo interativo até que o pesquisador aceite que a definição abstrata dada para o seu tema ou contexto, seja por ele aceita como a representação do contexto objetivo.

Assim foram definidos os eixos e palavras-chaves, conforme apresentado na Tabela 1 - Eixos de Pesquisa e suas palavras chave (Seção 3 - Referencial Teórico - 3.1 Gestão de Docentes em Instituições de Ensino Superior), condições necessárias mas não suficientes para o desenvolvimento da pesquisa.

O processo de busca iniciou-se a partir de uma expressão boleana, representada pela Figura 2 - Expressão Booleana Palavras-chave (Seção 4 Resultados - 4.1 Seleção do Portfólio Bibliográfico), que contém as palavras-chave alinhadas a cada eixo, a pesquisa foi realizada nas bases de dados da CAPES, conforme apresentado na Tabela 2 - Artigos Brutos Selecionados por Base de Dados (também apresentada na Seção de Resultados).

Inicialmente foram identificados 3.379 artigos que passaram a constituir o "portfólio bruto de artigos. A seguir, através da filtragem realizada com o uso do gerenciador bibliográfico EndNote X7, foram realizadas filtragens para remover os artigos duplicados. Foram eliminados 329 artigos repetidos e, num segundo momento foi realizada a filtragem pela leitura do título de cada um dos 3.050 artigos, quando foram eliminados mais 2.669 artigos que, no entendimento dos pesquisadores não estavam alinhados com o tema, reduzindo o Banco de Artigos Brutos para 381 artigos. 
Estes 381 artigos foram analisados quanto a sua representatividade científica quando então foram aceitos 185 artigos, a partir dos quais, foi composto o Banco de Artigos Não Repetidos, com Título Alinhado e Reconhecimento Científico. Estes artigos foram submetidos à leitura dos resumos que, resultou em 71 artigos considerados alinhados ao tema.

Os 196 artigos restantes, foram submetidos à uma reanálise em termos dos seus autores e ano de publicação tendo sido aceito para releitura os que haviam sido publicados em até dois anos, mais os artigos que tinham seus autores no banco de autores gerando assim o Banco de Artigos com Reconhecimento Cientifico Potencial. Neste processo, 62 artigos foram selecionados para a leitura do resumo, dos quais apenas 15 foram considerados alinhados ao tema.

Como resultado deste processo foram somados aos 71 artigos com reconhecimento científico confirmado os 15 artigos com menos de dois anos de publicação, totalizando 86 artigos que passaram pela etapa seguinte que é a realização da sua leitura integral.

Destes 86 artigos, apenas 37 estavam disponibilizados na internet, os demais foram descartados. Após a leitura dos 37 artigos na integra, 16 foram considerados alinhados ao tema, sendo classificados como Artigos Primários do Portfólio Bibliográfico.

Por fim, para garantir que nenhum artigo, com reconhecimento científico e alinhado ao tema, tenha ficado fora do PB, foi realizado o Teste de Representatividade do Portfólio Bibliográfico, no qual é realizado analisando as referências bibliográficas dos artigos que compõem o PB, e repetindo as mesmas etapas realizadas para a identificação do Portfólio Bibliográfico potencial.

Dos 16 artigos do PB, foram identificados 115 artigos denominados Artigos das Referências do Portfólio Bibliográfico que, após serem submetidos a todo o processo de filtragem, resultaram na identificação de 2 artigos alinhados ao tema. Estes, foram incorporados ao Portfólio Bibliográfico Final (PB) representado na Tabela 3 - Portfólio Bibliográfico (Seção 4 Resultados - 4.1 Seleção do Portfólio Bibliográfico) totalizando por 18 artigos.

Com a aplicação do instrumento de intervenção utilizado - o ProKnow-C, foi possível: 1) Selecionar um portfólio bibliográfico de artigos com reconhecimento científico e alinhado ao tema, segundo as percepções s pesquisadores; e 2) Realizar a análise bibliométrica no portfólio identificando que o periódico de destaque no PB é o Journal of Teacher Education com 3 artigos publicados, que o autor Lindblom-Ylänne, S. é destaque com 2 artigos 
publicados no PB e 1 artigo publicado nas suas Referências, o artigo The impact of training of university teachers on their teaching skills, their approach to teaching and the approach to learning of their students é o mais representativo do PB com 444 citações no Google Acadêmico, que as palavras-chave que mais se destacaram foram: Teacher com incidência em 17 artigos dos 18 que integram o PB, University com incidência em 16 artigos, Performance, Evalu* e Management que aparecem em 15 artigos e ainda que, as universidades que mais possuem pesquisadores no PB são: Islamia University of Bahawalpur, do Paquistão, com quatro pesquisadores, e a Faculty of Technology, University of Tlemcen da Argélia com 3 pesquisadores.

Diante dos resultados acima e, registrados na Seção 4 - Resultados, é possível evidenciar que o objetivo desta pesquisa foi alcançado, uma vez que permitiu aprofundar o conhecimento dos pesquisadores sobre o tema Gestão de Docentes em Instituições de Ensino Superior, com o intuito de conhecer as mais relevantes produções científicas internacionais sobre o assunto, seus autores, periódicos e palavras-chave.

Além disso, o Portfólio Bibliográfico, produto final deste trabalho, possibilitou a geração de conhecimento sobre o tema que servirá para orientação de futuros trabalhos.

Como recomendações aos futuros estudos sobre o tema sugere-se que, para o portfólio bibliográfico representativo do estado da arte do tema Gestão de Docentes em Instituições de Ensino Superior, segundo a visão do tema pelos pesquisadores, seja realizada uma análise do conteúdo buscando oportunidades e potencialidades inseridas em seu contexto.

\section{REFERÊNCIAS}

ALAVI, M.; CARLSON, P. A review of MIS research and disciplinary development. Journal of Management Information Systems, p. 45-62, 1992. ISSN 0742-1222.

BEHN, R. D. (2003). Why Measure Performance? Different Purposes Require Different Measures; Public Administration Review; September/ October; Vol 63, No. 5.

BROWN, W. O. Sources of funds and quality effects in higher education. Economics of Education Review 20 (2001) 289-295. Accepted 21 January 2000.

CASTLE, S.; FOX, R. K.; SOUDER, K. O'H. Do Professional Development Schools (PDSs) make a difference?: A comparative study of PDS and non-PDS teacher candidates. Journal of Teacher Education, Vol. 57, No. 1, January/February 2006 65-80. 
DAY, C.; ELLIOT, B.; KINGTON, A. Reform, standards and teacher identity: Challenges of sustaining commitment. Teaching and Teacher Education 21 (2005) 563577.

ENSSLIN, L.; ENSSLIN, S. R. (2007). Orientações para elaboração dos artigos científicos do LabMCDA-C [Apostila da disciplina Avaliação de Desempenho do Programa de Pósgraduação em Engenharia de Produção da Universidade Federal de Santa Catarina].

Florianópolis. UFSC.

ENSSLIN, L.; GIFFHORN, E.; ENSSLIN, S. R.; PETRI, S. M.; VIANNA, W. B. Avaliação do Desempenho de Empresas Terceirizadas com o Uso da Metodologia Multicritério de Apoio à Decisão - Construtivista. Revista Pesquisa Operacional, V.30, No. 1, p. 125 - 152, Janeiro a Abril de 2010. Versão impressa ISSN 0101-7438 / versão online ISSN 1678-5142.

ENSSLIN, S.R.; ENSSLIN, L.; MOREIRA, A. C. S.; PEREIRA, V.L.V.. Evidenciação do estado da arte da avaliação da segurança do trabalho em empreendimentos da construção civil. Interciencia, Vol.39, No. 1, pp. 16-23, jan 2014.

EVANS, L.; HOMER, M.; RAYNER, S. Professors as Academic Leaders: The Perspectives of "the Led". Educational Management Administration \& Leadership 2013 41: 674.

F. VERNADAT, F.; SHAH, L.; ETIENNE, A.; SIADAT, A.. VR-PMS: a new approach for performance measurement and management of industrial systems; International Journal of Production Research, 2013; Vol. 51, Nos. 23-24, 7420-7438, http://dx.doi.org/10.1080/00207543.2012.752593.

FIGUEIRA, J.; GRECO, S.; EHRGOTT, M. Multicriteria Decision Analysis: State of the Art Survey; Springer, 2005.

GIBBS, G.; COFFEY, M. The impact of training of university teachers on their teaching skills, their approach to teaching and the approach to learning of their students. Active Learning in Higher Education 1, Mar 2004.

GUEDES, V.L.S., BORSCHIVER, S. Bibliometria: uma ferramenta estatística para a gestão da informação e do conhecimento, em sistemas de informação, de comunicação e de avaliação científica e tecnológica. In: ENCONTRO NACIONAL DE CIÊNCIA DA INFORMAÇÃO, 6. 2005. Salvador. Anais... Salvador, 2005. p. 1-18.

GIL, A. C. Métodos e técnicas de pesquisa social. 6. ed. São Paulo: Atlas, 2008.

HALACHMI, E. (2005); Performance Measurement is Only One Way of Managing Performance; International Journal of Productivity and Performance Management - IJPPM; Vol. 54, No. 7, p. 502-516.

HENDERSON, C.; TURPEN, C.; DANCY, M.; CHAPMAN, T. Assessment of teaching effectiveness: Lack of alignment between instructors, institutions, and research recommendations. Physical Review Tópicos Especiais. 19, February, 2014. 
HUANG, Y.-C., LIN, S.-H. (2014). Assessment of Charisma as a Factor in Effective Teaching. Educational Technology \& Society, 17 (2), 284-295, 4 June, 2013.

KENNEDY, M. M. Knowledge and vision in teaching. Journal of Teacher Education, v. 57, n. 3, p. 205-211, 2006.

LABAREE, D. F. On the nature of teaching and teacher education: Difficult practices that look Easy. Journal of Teacher Education 1, maio 2000.

MEGNOUNIF, A.; KHERBOUCHE, A.; CHERMITTI, N. Contribution to the Quality Assessment in Higher Education_The Case Study of the Faculty of Technology, Tlemcen, Algeria. 6th International Forum on Engineering Education (IFEE 2012).

MORAES, L., GARCIA, R., ENSSLIN, L., DA CONCEIÇÃO; DE CARVALHO, S. (2010). The multicriteria analysis for construction of bench markers to support the Clinical Engineering in the Healthcare Technology Management. European Journal of Operational Research, v. 200, p. 607-615.

PAGE, D. Recruitment and Transition of Construction Lecturers in Further Education: The Perspective of Middle Managers. Educational Management Administration \& Leadership 2013 41: 819, originally published online 11, July 2013.

PAO, M. L. Global and local collaborators: a study of scientific collaboration. Information processing \& management, v. 28, p. 99-109, 1992.

PARPALA, A.; YLÄNNE, L. S. University Teachers' Conceptions Of Good Teaching In The Units Of High-Quality Education. Studies in Educational Evaluation 33 (2007) 355370 .

RASHEED, M. I.; ASLAM, H. D.; YOUSAF, S.; NOOR, A. A critical analysis of performance appraisal system for teachers in public sector universities of Pakistan: A case study of the Islamia University of Bahawalpur (IUB). African Journal of Business Management v.5, n.9, p. 3735-3744, 4 May, 2011.

REID, I. C. Reflections on using the Internet for the evaluation of course delivery. University of South Australia, Underdale Campus, Holbrooks Road, Underdale 5032, South Australia, Australia. Accepted 27 July 2001.

RUEDA B.M.. La Evaluación del Desempeño Docente en la Universidad. The Evaluation of Teacher Perfomance at the University. Revista electrónica de investigación educativa. Publicado en 22 de octubre de 2008.

RICHARDSON, R. J. Pesquisa social, métodos e técnicas. São Paulo: Atlas, 1999. Pesquisa social, métodos e técnicas. 3. ed. São Paulo: Atlas, 2008. 
RYHAMMAR, L.; ANDERSSON, A. L. Relations between university teachers' assessed degree of creativity and productivity and views regarding their organization. Journal of Creative Behavior. Vol. 35 Number 3 Third Quarter 2001.

SANTOS, R. N.; ALCÂNTARA-ELIEL, R.; ELIEL, O. A ciência e o novo estado do conhecimento: a contribuição da ciência da informação. Enc. Bibli: R. letr. Bibliocon. Ci. Inf., n. 22, p. 16-29, 2006.

STEWART, M. Making sense of a teaching programme for university academics: Exploring the longer-term effects. Teaching and Teacher Education, v. 38, 2014.

TASCA, J. E., ENSSLIN, L.; ENSSLIN, S. R.; ALVES, M. B. M. (2010). An approach for selecting a theoretical framework for the evaluation of training programs. Journal of European Industrial Training, v.34, n.7, p. 631-655.

TEJEDOR T.F.J.; MELIÁ, J. M. J.. University Teacher's Evaluation in Spain. Revista electrónica de investigación educativa. Publicado em 15 de octubre de 2008. 